\title{
SWIFT J1753.5-0127: a surprising optical/X-ray cross-correlation function
}

\author{
Martin Durant \\ Instituto de Astrofísica de Canarias, La Laguna, E38205 Tenerife, Spain \\ durant@iac.es \\ Poshak Gandhi \\ RIKEN Institute of Physical and Chemical Research, 2-1 Hirosawa, Wakoshi, Saitama, \\ Japan \\ Tariq Shahbaz \\ Instituto de Astrofísica de Canarias, La Laguna, E38205 Tenerife, Spain \\ Andy Fabian \\ Institute of Astronomy, Madingley Road, Cambridge, UK \\ Jon Miller \\ University of Michigan, 500 Church Street, Ann Arbor MI, USA \\ V. S. Dhillon \\ Department of Physics and Astronomy, University of Sheffield, Sheffield S3 7RH, UK \\ and \\ Tom R. Marsh \\ Department of Physics, University of Warwick, Gibbet Hill Road, Coventry CV4 7AL, UK
}

\begin{abstract}
We have conducted optical and X-ray simultaneous observations of SWIFT J1753.5-0127 with RXTE and ULTRACAM, while the system persisted in its relatively bright low/hard state. In the cross-correlation function (CCF), we find that the optical leads the X-rays by a few seconds with a broad negative peak, and has a smaller positive peak at positive lags. This is markedly different from what was seen for the similarly interesting system XTE J1118+480, and the first time such a correlation function has been so clearly measured. We suggest a physical scenario for its origin.
\end{abstract}


Subject headings: binaries: individual (SWIFT J1753.5-0127)

\section{Introduction}

X-ray and optical emission from astrophysical objects are produced by very different means, with different energetics and time-scales. X-ray binaries are prolific sources of both, with measurable rapid variability. The emission modes cannot be wholly independent, so by comparing their inter-connection, we can learn about the physical conditions from the main emission region: the inner disc around a neutron star or black hole.

Whilst high time resolution $(\leq 1 \mathrm{~ms})$ for X-ray observations has been achieved from the very earliest observations, the photon rates for most sources prevented statistically significant timing work. In the optical domain, exposure times in the ms domain have only become possible relatively recently, with low enough noise and dead-time and high enough quantum efficiency (i.e., CCDs as opposed to photometers) to achieve good signal-to-noise ratios per exposure. The final problem has been simply to schedule simultaneous X-ray and optical observations.

The black hole X-ray Transient (XRT) SWIFT J1753.5-0127 is a system which has been of great interest recently following its outburst episode and detailed observations with the SWIFT satellite. First discovered by the SWIFT/BAT (Burst Alert Telescope; Palmer et al., 2005) in 2005, pointed $\gamma$-ray, X-ray, UV, optical and radio observations all detected a new bright source at this location (Morris et al., 2005; Still et al., 2005; Halpern 2005; Fender et al., 2005). Following the early report of a $0.6 \mathrm{~Hz}$ quasi-periodic oscillation (QPO; Morgan et al. 2005; Ramadevi \& Seetha, 2005), persistent for some time after the bursting episode, we applied to observe the system simultaneously in X-rays and optical as it faded 1 . The source was still relatively bright however, especially in the optical, at the time of our project. Following the burst, Cadolle-Bel et al. (2007) measured the spectrum from radio up to $600 \mathrm{keV}$, and Miller et al. (2006) showed spectroscopically that a disc reaching down to small radii was likely.

Further details of our observational campaign, including spectroscopy, longer-term multiband optical photometry and detailed periodogram analysis are to be published separately in Durant et al. (2008), and an analysis of the long-term R-band variability and orbit-like modulation are presented in Zurita et al (2008). Here, we intend to make the minimum number of processing steps and assumptions to obtain the X-ray/optical CCFs a unique

\footnotetext{
${ }^{1}$ ESO and RXTE observation IDs 079.D-0535 and 93119-02-02-00, respectively.
} 
phenomenological hint of black hole accretion physics.

\section{Observations}

SWIFT J1753.5-0127 was observed for 53.6 min on 13 Jun 2007 with the Rossi X-ray Timing Explorer (RXTE), which provides very high timing resolution $(\sim 1 \mu \mathrm{s})$, reasonable energy resolution $(\sim 1 \mathrm{keV})$ and very high effective area in the $2-100 \mathrm{keV}$ range. During this observation, three of the units of the Proportional Counting Array (PCA, Bradt et al. 1993) were active. We do not consider here the data of the HEXTE or ASM instruments, where the count rates were much lower. The $2-20 \mathrm{keV}$ flux was $1.6 \times 10^{-9} \mathrm{erg} \mathrm{cm}^{-2} \mathrm{~s}^{-1}$ (with standard background subtraction).

We produced four light-curves of the data using the FTOOLS task seextrct with standard good time filtering and standard settings. The bands were selected purely by splitting the energy channels into four equal segments. We have not attempted to calibrate these energy cuts exactly: the rough general mapping of channel number to energy at heasarc.nasa.gov/docs/xte/e-c_table.html is sufficient for our uses. The variability was at the $\sim 40 \%$ level (in which Poissonian noise is significant).

SWIFT J1753.5-0127 was simultaneously observed with ULTRACAM, mounted on VLT/3 (Melipal) telescope. Of the 75 min observation, 50 min were simultaneous with the RXTE observation. ULTRACAM is an instrument employing dichromatic beam splitters, frame-transfer CCDs and a GPS-based timing system in order to be able to make simultaneous multi-wavelength light-curves at very high time resolution, up to $300 \mathrm{~Hz}$ (Dhillon \& Marsh, 1999; Dhillon, 2007). We used two small windows on each CCD (one for the source of interest, one for a local standard), with exposure times of $140 \mathrm{~ms}$ (resulting in a duty cycle of $142 \mathrm{~ms}$ ). Here we note that conditions were fairly poor, with thin cloud causing transparency and seeing variations, mostly on timescales longer than $10 \mathrm{~min}$. Fluxes were extracted by aperture photometry with a variable aperture size scaled to the FWHM of the reference star on each image. This enables some optimization for signal to noise under the variable conditions. Short-term variability in the optical band was at the $\sim 10 \%$ level. 


\section{Cross-correlation}

We calculated the CCFs of each of the X-ray light-curves with each optical light-curve produced above 2 The results can be seen in Figure 1. The curves have been scaled such that one unit equals the typical difference between one point and the next - it is therefore a simple measure of the random ('white') noise, as seen in the cross-correlation. The scale is therefore a simple measure of significance.

Whilst the functions are clearly very similar between optical bands (except that the g' band is much noisier), there is a marked difference between the behavior seen with X-ray energy range:-

- For the 0-63 channel range, which in Epoch 5 corresponds roughly to $2-27 \mathrm{keV}$, a strong signal is seen at negative lags (optical leading X-rays), followed by a somewhat weaker inverted signal. The width of these signals is of the order $10 \mathrm{~s}$. There is further, less significant structure in the CCF apart from the two main features mentioned. This could hint at some oscillatory interaction between the optical and X-ray emission.

- In the $64-127$ channel range $(\sim 27-55 \mathrm{keV})$, a weaker but significant signal is seen as for $0-63$, but notably narrower in its response.

- For the two remaining curves at higher energies $(\sim 55-118 \mathrm{keV})$, no significant signal is seen at all.

In every CCF, no further significant features are seen for $|\delta t|>30 \mathrm{~s}$. The g' band may show a broader response than the r' band. With the poorer data in the former, we regard this as merely a suggestion.

Significantly, the cross-correlations here do not change noticeably during the simultaneous window, when constructed for sections of the data. This is despite the window length ( $\sim 50 \mathrm{~min}$ ) being significant compared to the orbital-like modulation period of $3.2 \mathrm{~h}$ (Zurita et al. 2008).

To investigate what features of the light curves are responsibe for the CCFs in Figure1, we performed the following check. For each of the $1 \%$ brightest bins of the X-ray total light curve, we averaged windows of the optical light curve centred on these bins. The resulting average looks like the solid line in Figure1 (the X-ray light curve is, of course, dominated in

\footnotetext{
${ }^{2}$ The light-curves themselves, periodograms and auto-correlations will be published separately (Durant et al., 2008).
} 
terms of counts by the lower energy range). Conversely, for the average of windows of the optical light curve centred on the $1 \%$ faintest $\mathrm{X}$-ray bins, the average function is again like the solid curve, but with the y-axis flipped. In other words, the optical light curve, some seconds before the X-ray, tends to be going in the opposite direction; yet the optical a few seconds after the X-ray tends to be going in the same direction, somewhat weaker. Since neither light curve can be decomposed into discreet events or flares, this relationship only comes out over an average of the whole light curve.

We constructed minimum-assumption spectra from the data, to investigate further which component is varying. Figure 2 shows the count rate per energy channel for the $\sim 10 \%$ brightest and faintest bins, along with the spectrum for all bins. One sees that from the lowest energies measured $(\sim 2 \mathrm{keV})$ to about Channel $70(\sim 30 \mathrm{keV})$, the spectrum maintains the same form, fluctuating by about a factor of 2 . Up to Channel $130(\sim 60 \mathrm{keV})$, some variation is seen, but none at higher energies. Thus it is not surprising that the higher energy bands above do not correlate at all with the optical. Note that the count-rates are clearly much lower in this upper energy range, and furthermore that the background rate becomes dominant. It is not surprising, therefore, that Figure 1 shows no relationship in comparison to the white noise above $\sim 60 \mathrm{keV}$. There are, however, still some counts above the background for this hard source. We compared the ratio of the sum of background-subtracted counts in the High and Low curves above and below channel 127, and find that they are very similar, perhaps the high-energy part showing an even higher ratio (i.e., it varies more). In general, it seems that the spectrum varies in amplitude only and not in shape. The modelling of background spectra has been a rapidly changing calibration issue 3 , however, so we regard this as suggestive. We used the latest calibration data available in April/May 2008.

\section{Discussion}

Very few X-ray/optical CCFs are recorded in the literature, mostly of systems in low states, where the optical lags the X-rays, and is assumed to be the reprocessing signature, possibly from the large inner radius of a truncated accretion disc. This can be used for tomography of the disc and companion by tracking the lag evolution with spectral range and orbital phase (see particularly Hynes, 2005). The dearth of cross-correlations is in good part due to the logistics of arranging simultaneous observations with the few instruments capable, within the short window following an outburst. In this respect, SWIFT J1753.5-0127 has been unique, by persisting in its low/hard state (relatively bright) for years after outburst

\footnotetext{
${ }^{3}$ http://www.universe.nasa.gov/xrays/programs/rxte/pca/doc/bkg/bkg-2007-saa/
} 

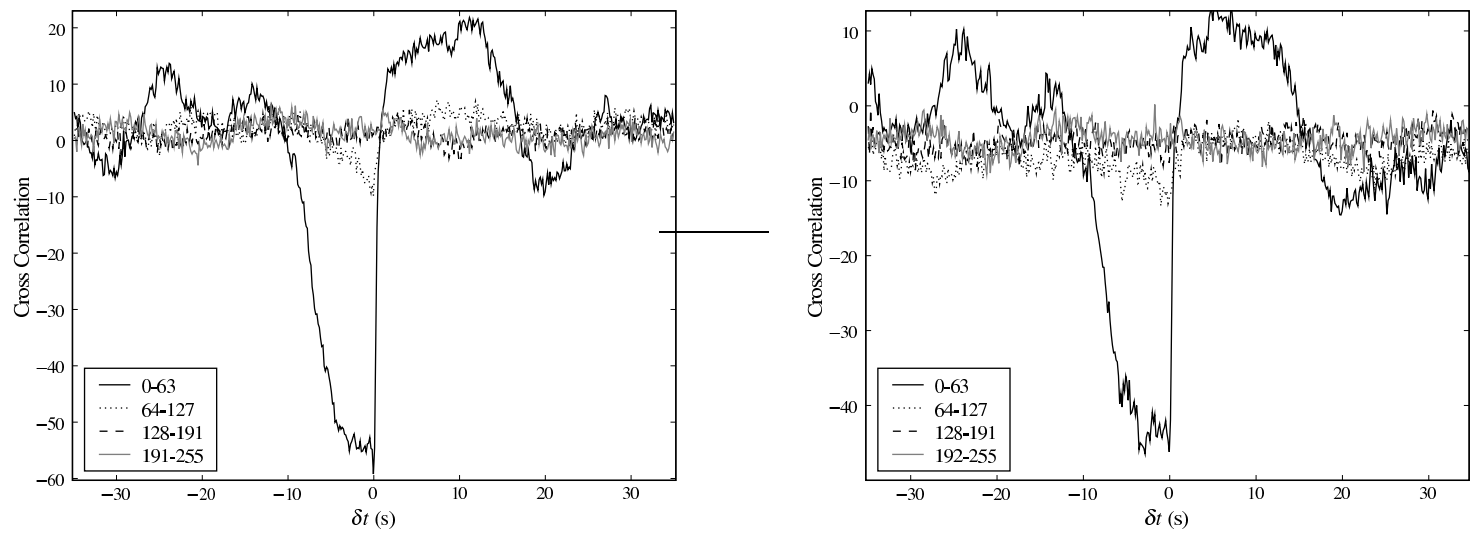

Fig. 1.- Cross correlation of RXTE/PCA and ULTRACAM light-curves of SWIFT J1753.5-0127. $\delta t<0$ represents optical light arriving earlier than X-rays. Each plot shows four X-ray energy ranges, by PCA channel number, for the r' band (left) and for the g' band (right). Scaling is relative to the noise in each CCF (see text).

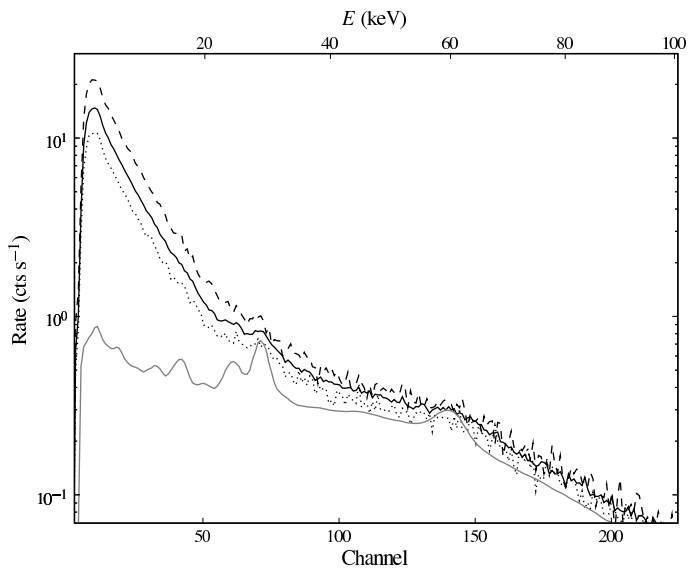

Fig. 2.- Counts spectrum extracted for the RXTE/PCA observation of SWIFT J1753.5-0127, uncalibrated: mean spectrum (solid line), high count rate bins (dashed line) and low count rate bins (dotted line). Note the logarithmic vertical scale. For comparison, we also plot the estimated background spectrum (gray), see text. Note how the background curve approaches but does not exceed the average spectrum. 
(Zurita et al. 2008). We may find that similar relations exist in other systems, which have gone undetected for technical reasons. Notably, Hynes et al. (2006) report the optical lagging the X-rays in this system by $\delta t<10 \mathrm{~s}$ during outburst, so clearly it was in some different mode during our observations.

Most typically, one expects X-ray flaring to occur in the innermost, hottest regions, and power optical emission by reprocessing of the X-ray flux further out in the optically thick accretion disc, or on the surface of the companion. This picture is a natural consequence of the typical ADAF model geometry, where the disc truncates at a large inner radius, inside of which material is hot and low-density (the ADAF itself), where X-rays are produced. One would expect from this a CCF positive response with a steep rise and slower fall. The positive part of our CCFs do not show this, so simple reprocessing does not dominate over the positive lag region.

Alternatively, X-ray emission may be from a magnetically driven corona around the dense accretion disc (ADC), where reconnection produces energetic particles, and the energy release is dominated by hard X-rays, from the up-scattering of photons by these highly energetic coronal particles. The different energy outputs can thus be coupled and interrelated in a complex manner, e.g., the jet-disc coupling model of Malzac et al. (2004). The corona can form a self-limiting feedback system (see e.g., Uzdensky \& Goodman 2007, 2008) wherein particles can evaporate from the disc to the corona or condense back to the disc, and the reconnection rate is determined by the density of the corona and magnetic loop movement. Reconnection occurs preferentially in a marginally collision-less coronal medium where free magnetic and particle kinetic energies are comparable, which results in a stable equilibrium for a given parameter set (energy transfer rate, sheering etc.).

If the emission is composed of localized micro-flares (flickering), each flare might proceed thus: between flares, a patch of the accretion disc cools, and particles from the corona are able to condense; as the coronal density drops, it becomes less collisional and magnetic energy dominated towards the marginally collisionless state, where particle density cannot inhibit magnetic reconnection. At a critical point, cool X-rays flash at the moment of reconnection, decreasing the stored magnetic energy; the disc is heated again, and the coronal particle population replenished by evaporation. Thus, the X-ray emission of the micro-flare is timed after a period of condensation, when the optical emission would be decreasing as more particles are shielded behind cyclotron absorption (indeed, free particles in the corona may also radiate by cyclotron/curvature); immediately afterwards, a population of corona particles and possibly the surface area of the disc patch is larger, so the optical emission is enhanced again. Only a fraction of the released X-rays and accelerated particles heat the disc, most escape or are emitted at higher energies. The self-regulating aspect, in which 
the X-ray luminosity acts in the opposite sense and reverses a dip in the optical; magnetic energy driving and disc evaporation/condensation do seem consistent with our CCF. How magnetic field is transmitted from the disc to the corona is not known.

This agrees roughly with the model by Fabian et al. (1982), where emission is controlled by the growing and contraction of optically thick, cyclotron-emitting plasma clouds. This model fitted well with the early measurements of GX 339-4 (see below). Since the energy emitted by cyclotron depends on the electron number and magnetic energy density, as field is expelled from dense regions, the optical emission would decrease while the energy available for reconnection increases. If the cyclotron emission is predominantly optically thick, then the emitted luminosity in the optical depends on the surface area of these clouds - as they contract and expel magnetic field to the more tenuous, hot medium, the optical emission decreases as the X-ray emission increases, they are naturally anti-correlated.

If the optical emission is not, then, predominantly jet-like, then there is no longer a reason to expect radio emission to correlate with luminosity: Cadolle-Bel et al. (2007) noted (confirmed by Soleri et al. 2007) that the radio emission was unusually low, and that its synchrotron-like spectrum fell below the optical emission. Furthermore, reprocessing of the emitted X-rays will be affected by the acceleration of plasma in magnetic reconnection events. If there is mildly relativistic bulk motion away from the denser matter, then the reprocessing would be weakened and show a different time-response (Beloborodov, 1999).

In the recent work by Liu et al (2007), they show that SWIFT J1753.5-0127 in particular, and also GX 339-4 can be modelled as a cool inner disc where thermal conduction and Compton cooling are important in this disc's interaction (condensation and evaporation) with the surrounding low-density corona. They do not specifically consider timescales and driving in their model, but their model is at least consistent with the results here and similar to the picture presented above, and to the dynamic picture of Fabian et al. It will be interesting to see further development of their model.

In our source, the high-energy emission dominates the total luminosity (up to INTEGRAL energies, see e.g., Cadolle-Bel et al. 2007). The picture is, therefore, of emerging magnetic flux from the disc, releasing its energy in the corona in a self-regulating way. Optical emission is from the dense, hot, magnetically active disc (by cyclotron/synchrotron) and by particles in the corona (by cyclotron and curvature). We believe this scenario accounts qualitatively for what is seen, but is short of a proof. 


\subsection{Comparison with XTE J1118+480 and GX 339-4}

Two objects that have been extensively studied, including simultaneous X-ray/optical projects, are XTE J1118+480 and GX 339-4.

XTE J1118+480 seems initially very similar in many characteristics to SWIFT J1753.5-0127: persistent low/hard state, high galactic latitude, X-ray spectral and timing characteristics.

Kanbach et al. (2001) found that the optical emission lags the X-rays by a small amount $(\sim 0.5 \mathrm{~s})$, but there is an interesting 'precognition dip' in the CCF which is difficult to explain. These features may be qualitiatively similar to our CCF, but in our case, the main feature is a strong anti-correlation, optical before X-ray. The alternative view would be that we see the same precognition dip and response signal, but with very different intensities. Interestingly, they find that the dip is stronger for longer wavelength optical data, which would also fit our data (with the caveat on the quality of the g' band observations above). It was to describe this system that Malzac et al. (2004) developed their jet-disc coupling model.

In the earliest such measurement made that we are aware of, Motch et al (1983) derived the X-ray/optical CCF for GX 339-4. From a very short simultaneous observation window (96s), they suggested a optical-leading anti-correlation, but only at energies $E<13 \mathrm{keV}$. It was in this context that the model of Fabian et al. (1982, above) was fairly successful. The CCF was not independently confirmed, but appears similar to our work. Later, Gandhi et al. (2008 in prep.) repeated these measurements over a longer time base-line for the source presumably in quiescence, and found that the CCF similar to XTE J1118+480, with the strongest feature a weak positive peak showing a slight lag of optical behind X-rays (by $\sim 0.2 \mathrm{~s}$ ), but the peak has a markedly different shape with a shallow rise and steep fall, followed by negative correlation in the $1-3 \mathrm{~s}$ lag range.

These comparisons are suggestive that the CCF we find is symptomatic of the accretion mode in our object, at the time of observation.

\subsection{Conclusions}

Notwithstanding the technical difficulties of constructing X-ray/optical cross-correlations, of the few capable instruments and simultaneous scheduling, this work presents the functions for SWIFT J1753.5-0127 which challenge our understanding of the physical processes in the immediate vicinity of a black hole. We find a strong anti-correlation, with the optical preceding the X-rays on time-scales of $1-10 \mathrm{~s}$. This demonstrates that there exists a causal link between the optical and X-rays, aside from simple reprocessing, and detailed dynamical modelling will be required to describe the system more fully. 
Acknowledgements: MD and TS are funded by the Spanish Ministry of Science. PG is a Fellow of the Japan Society for the Promotion of Science (JSPS). ULTRACAM was designed and built with funding from PPARC (now STFC), and used as a visiting instrument at ESO Paranal, and RXTE is operated by NASA. Partially funded by the Spanish MEC under the Consolider-Ingenio 2010 Program grant CSD2006-00070: "First Science with the GTC" (http://www.iac.es/consolider-ingenio-gtc/).

\section{REFERENCES}

Beloborodov, A., 1999, ApJ, 510, L123

Bradt, H., Rothschild, R., Swank, J., 1993, A\&AS, 97, 355

Cadolle Bel, M., Ribó, M., Rodriguez, J., 2007, ApJ, 659, 549

Dhillon, V. \& Marsh, T., 1999, New Astronomy Reviews, 45, Issue 1-2, p. 91

Dhillon, V., Marsh, T., Stevenson, M, Atkinson, D., Kerry, P., et al. 2007, MNRAS, 378, 825

Durant, M., Gandhi, P., Shahbaz, T., H. Peralta., H., 2008, MNRAS, submitted

Fabian, A., Guilbert, P., Motch, C., Ricketts, M., Ilovaisky, S., Chevalier, C., A\&A, 111, L9

Fender, R., Garrington, S., Muxlow, T., 2005, ATel, 558

Gandhi et al., 2008 in preparation

Halpern, J., 2005, ATel, 549

Hynes, R., Haswell, C., Cui, W., Shrader, C., O’Brien, K., Chaty, S., Skillman, D., Patterson, J., Horne, K., 2003, MNRAS, 345, 292

Hynes, R., 2005, ASPC, 330, 237

Hynes, R., 2006, AIPC, 840, 88

Kanbach, G., Straubmeier, C., Spruit, H., Belloni, T., 2001, Nature, 414, 180

Liu, B., Taam, R., Meyer-Hofmeister, E., Meyer, F., 2007, ApJ, 671, 695

Malzac, J., Merloni, A., Fabian, A., 2004, MNRAS, 351, 253

Miller, J., Homan, J, Miniutti, G., 2006, ApJ, 652, L113 
Morgan, E., Swank, J., Markwardt, C., Gehrels, N., 2005, ATel, 550

Morris, D., Burrows, D., Racusin, J., Roming, P., Chester, M., Verghetta, R., Markwardt, C., Barthelmy, S., 2005, ATel, 552

Motch, C., Ricketts, M., Page, C., Ilovaisky, S., Chevalier, C., 1983, A\&A, 119, 171

Palmer, D., Barthelmey, S., Cummings, J., Gehrels, N., Krimm, H., Markwardt, C., Sakamoto, T., Tueller, J., 2005, ATel, 546

Ramadevi, M., Seetha, S., 2007, MNRAS, 378, 182

Still, M., Roming, P., Brocksopp, C., Markwardt, C., 2005, ATel, 553

Uzdensky, D., Goodman, J., 2007, MmSAI, 78, 403

Uzdensky, D., Goodman, J., 2008, ApJ submitted (astro-ph/0803.0337)

Zurita, C., Durant, M., Torres, M., Shahbaz, T., Cesares, J., 2008, ApJ, in press (astro$\mathrm{ph} / 0803.2524)$ 